\title{
Round robin testing of synthetic fibre ropes for application in marine renewable energy
}

\author{
F. Khalid, P. Halswell, P.R. Thies \& L. Johanning \\ College of Engineering, Mathematics and Physical Sciences, University of Exeter, Penryn Campus, UK
}

P. Davies \& Nicolas Lacotte

Materials \& Structures group, IFREMER Brest Centre, France

\begin{abstract}
The unique design requirements of mooring systems in Marine Renewable Energy (MRE) installations require detailed numerical and empirical investigations as well as offshore experience to adopt and certify fibre ropes in the marine renewable energy industry. Laboratories provide a controlled environment for quick, inexpensive and repeatable testing compared to field deployment and enable a range of parameters to be studied. Uncertainties may be introduced in the laboratory test results because of effects associated with individual facilities (such as instrumentation accuracy, control system and analysis methods) despite following the same protocol. This paper presents the outcomes of a round robin testing campaign conducted at two test facilities to conduct a comparative analysis by monitoring the implementation of the test program and analysing the results to highlight differences between facilities and suggest best practice for fibre rope testing in the MRE industry.
\end{abstract}

\section{INTRODUCTION}

Mooring systems of dynamic floating MRE devices are required to provide effective station-keeping whilst reducing the combined effects of metocean loads on the system. Conventional station-keeping solutions, composed of steel wire and chain systems, fail to provide the necessary compliance to minimise peak loads for a highly dynamic system (Flory, JF; Banfield, SJ; Ridge, 2016). This reduces system reliability, where a mooring component failure may lead to interruption of operations and incur high offshore repair costs.

In the MRE industry, there is a marked shift from conventional mooring methods to the use of synthetic fibre ropes. This is driven by the performance-related benefits associated to energy take-off and reliability (The Carbon Trust, 2011). Based on the success of synthetic fibre rope in previous MRE prototypes, new guidance available for offshore wind (ClassNK, 2012; DNV GL, 2018), wave (Det Norske Veritas (DNV), 2008; Khalid et al., 2019) and tidal energy (DNV-GL, 2015; IEC, 2013) provides recommendations on effective use of fibre rope mooring components.

Commonly used synthetic materials in mooring applications include polyamide (nylon) and polyester. In addition to possessing favourable mechanical properties such as a comparable Minimum Breaking Load (MBL), these synthetic mooring elements are an inexpensive alternative to conventional steel elements. Due to this reason, polyester has been widely adopted for deepwater moorings of offshore platforms for the past two decades (Davies et al., 2014). Due to its higher compliance, nylon displays an excellent shock load absorbing ability, which is a suitable characteristic for highly dynamic bodies such as MRE devices (Ridge et al., 2010). However, the axial stiffness of nylon is adversely effected by water ingress (Gordelier, 2016). Polyolefin is a less known material for mooring applications, however, with stiffness characteristics similar to nylon and polyester it could be a suitable material for synthesizing MRE mooring components.

To increase confidence in fibre ropes mooring solutions, the component physical properties and performance attributes must be thoroughly investigated. Existing methodologies for synthetic fibre rope mooring testing in MRE are primarily adopted from offshore oil and gas applications (Weller et al., 2015). The application of this knowledge to MRE systems is limited since the loading regimes at $O \& G$ platforms beyond 1,000 meters depth are significantly different from those experienced by MRE deployed at sites with depths up to hundreds of meters. Therefore, test methods need to be modified for improved performance characterisation. As field testing is associated with high risk and test cost, an alternative is testing individual components at purpose-built test facilities.

A limited number of test facilities, operated by rope manufacturers or research and academic institutes, are available for fibre rope testing (Davies et al., 2012). While providing a similar range of services, 
the test set-up and instrumentation at these facilities vary. Independent calibrations and certifications are regularly conducted at most facilities but no comparative study has been conducted, yet regarding the influence of test infrastructure on the performance characterization.

The commercialisation of MRE can be accelerated by streamlining the capabilities of test infrastructures in order to enhance their impact through Round Robin Testing (RRT). RRT is a monitoring tool that requires repeated implementation of the same test program adopted from standard guidance on multiple samples at the involved facilities. It can allow a direct correlation and quantification of the influence that individual test facilities have on the test outcomes.

This paper presents a RRT study investigating the suitability of established standard test procedures to characterize a hybrid fibre rope for MRE mooring component testing. The RRT campaign was conducted at two test facilities, namely the Dynamic Marine Component (DMaC) test facility at the University of Exeter and The Marine Structures laboratory at L'Institut Français de Recherche pour l'Exploitation de la Mer (IFREMER). The rope samples were characterised by the quasi-static and dynamic stiffness properties as well as the break loads and failure modes at each facility.

\section{METHODOLOGY}

\subsection{Test specimen}

The round robin test specimen was a EUROFLEX ${ }^{\circledR}$ rope (Figure 1), composed of a combination of polyester fibres with a blend of polyolefin (polymerized propylene). It is a white twisted 3-strand rope with a yellow marker yarn. The associated properties of the 3 - strand EUROFLEX ${ }^{\circledR}$ rope procured from Lankhorst (Lankhorst Ropes, 2020) are detailed in Table 1.

Table 1. Material properties of the fibre rope specimen.

\begin{tabular}{ll}
\hline Property & Description \\
\hline Rope reference & EUROFLEX® 3 Strand \\
Article number & 152.532 \\
Diameter & $32 \mathrm{~mm}$ \\
Weight & $59.5 \mathrm{~kg} / 100 \mathrm{~m}$ \\
Minimum breaking load & $168 \mathrm{kN}$ \\
\hline
\end{tabular}

Five spliced test samples were procured for each test facility with a length of $5 \mathrm{~m}$ from bearing to bearing. The diameter of each splice eye was approximately $0.6 \mathrm{~m}$ that allowed $2.5 \mathrm{~m}$ of unspliced rope for testing. The length of the samples tested at DMaC was reduced by resplicing to compensate for the additional elasticity of the rope to conduct successful load-tofailure tests.

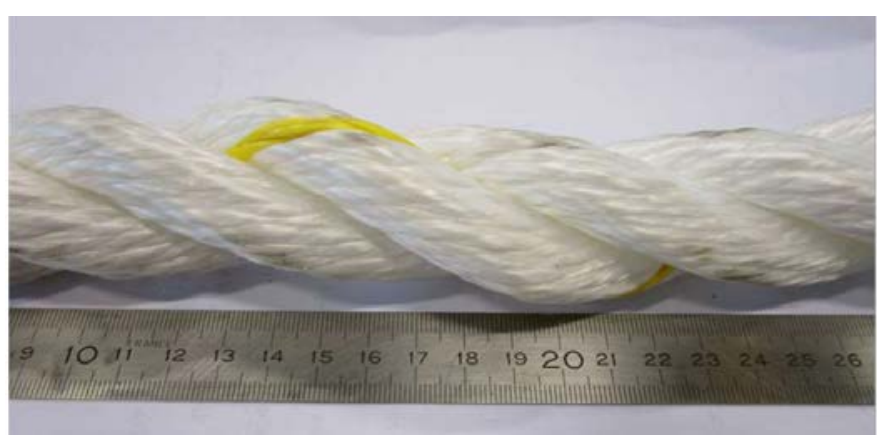

Figure 1. Test specimen for RRT: EUROFLEX® 3 Strand rope manufactured by Lankhorst Ropes.

\subsection{Test facilities and instrumentation}

The involved test facilities, DMaC and IFREMER, have a range of instrumentation that could contribute to differences in the implementation of the test plan. This includes the load cell, control system, data acquisition system and the established procedure to maintain ambient conditions.

\subsubsection{DMaC}

The DMaC machine comprises of a DSCC pancake load cell manufactured by Applied Measurement Ltd, UK (serial number 50317) with a full-scale linearity of $\pm 0.039 \%$.

An IP67-rated, WS12 draw-wire transducer manufactured by Applied Measurements was used to measure sample elongation during the bedding-in and dynamic stages of the test at a sample rate of $50 \mathrm{~Hz}$. The draw-wire transducer was recording using the NI cRIO 9022 and NI 9205 C-series module. With the transducer body clamped to the sample using a custom-made clamp, the end of the draw-wire was attached to the sample using a bungee cord (via an additional length of wire) to provide a gauge length greater than $1.2 \mathrm{~m}$.

$\mathrm{DMaC}$ has a synchronised control and data acquisition system, which enables both specified and measured values to be appended, at each time step, to a single results file. For the tests reported here, the axial load experienced by the main hydraulic cylinder and piston displacement were simultaneously logged at a sample rate of $50 \mathrm{~Hz}$. In addition to data logging, the $\mathrm{DMaC}$ data acquisition system was used to monitor piston displacement and axial load during the test setup, allowing the reference tension to be set prior to testing.

Piston displacement was measured using a LM10 linear encoder manufactured by RLS Merilna tehnika d.o.o., Slovenia; resolution of $0.05 \mathrm{~mm}$. The measurements are recorded using a National Instruments (NI) compact Reprogrammable Input Output (cRIO) 9022. Load measurements utilise a NI 9237 C-Series module and displacement measurements use a NI 9205 CSeries module.

At DMaC, samples are fully submerged in tap water throughout the test campaign. 


\subsubsection{IFREMER}

The load cell of the IFREMER machine is an AEP TC4 $300 \mathrm{kN}$ model that is calibrated annually by an external company.

Strain measurements were obtained from two wire displacement transducers placed at the two ends of the rope. They were mounted on an L-frame in order to be at the right height. At the fixed end of the rope the transducer was an ASM WS10-500mm displacement model. At the moving (piston) end an ASM WS10-1250mm model was used. The ends of their wires were fixed to the rope in the central section using elastic loops to calculate elongation. The two wire transducers were provided with supplier calibrations but both were checked manually before each test.

Piston displacement measurement was recorded throughout the tests, using an SCAIME wire transducer model PT5DC-40. The MTS "MultiPurpose Elite" software controls the piston movement and allows test sequences to be recorded. It also allows continuous recording of force, piston displacement, air and water temperatures and external displacement transducers throughout each test.

Data was recorded at two acquisition frequencies. A frequency of $1 \mathrm{~Hz}$, to provide a first overview of the test, and higher frequency $(5 \mathrm{~Hz})$ for the dynamic stiffness measurements. The stiffness values given in this report were obtained from the data recorded at 1 $\mathrm{Hz}$ unless otherwise stated.

AT IFREMER, the samples are sprayed with freshwater throughout the test except for the final ramp to failure.

\subsection{Test plan}

The RRT plan is based on ISO 18692:2007(E) (ISO, 2007 ) that provides guidance for testing polyester fibre ropes used for offshore station keeping of permanent or mobile floating structures. This standard is commonly used to develop the fatigue test procedure at DMaC and IFREMER.

The standard test plan is divided into Phase $\mathrm{A}$ and Phase B. The test duration of Phase A is 5 h 50 min $20 \mathrm{~s}$ and includes sample bedding-in, quasi-static and dynamic loading. Phase B is a linear ramp to failure under load control. Table 2 describes the steps of ISO 18692:2007(E) mapped to Phase A and Phase B.

Table 2. Description of constituent steps of ISO18692:2007(E) in Phase A and Phase B.

\begin{tabular}{lll}
\hline Phase & Steps & Description \\
\hline Phase A & 6,7 & Bedding in (static) \\
\cline { 2 - 3 } & 8 & Bedding in (dynamic) \\
\cline { 2 - 3 } & 9 & $\begin{array}{l}\text { Quasi-static loading } \\
\text { Dynamic loading }\end{array}$ \\
\hline Phase B & 10 & Load-to-failure \\
\hline
\end{tabular}

\subsubsection{Sample preparation}

Each sample was soaked in fresh water overnight prior to testing to account for the effect of moisture absorption on rope stiffness and strength. In accordance with ISO 18692:2007(E), the attachment points of the transducer body and wire were at least three times the rope diameter from the end of the eye splice on each side. Once the sample and transducers were installed in the test machine, two length measurements were recorded at the start of the test under a load equivalent to $2 \% \mathrm{MBL}(3 \mathrm{kN})$, recording:

- Total length, $\mathrm{L}_{\mathrm{T}}$

- $\quad$ Reference length, $L_{R}$

For $\mathrm{DMaC}, \mathrm{L}_{\mathrm{T}}$ is the eye-to-eye length between the shackles and $L_{R}$ is the gauge length at the reference tension. At IFREMER, $\mathrm{L}_{\mathrm{T}}$ is measured as the length between the pin centres and $L_{R}$ is measured as the distance between the loops of the two transducers at reference tension.

To reflect the intended application of the ropes, tests were performed with the ropes either fully submerged in water (DMaC) or continuously sprayed with water (IFREMER).

At the outset of Phase A, the sample is bedded-in to allow individual fibre components and the eye splices to achieve an optimal state. This results in the rope reaching a stable and repeatable condition for further testing.

It is recommended that the rope is bedded-in by static and dynamic loading. For the static bedding-in, the rope is extended to a load of $50 \%$ of the MBL at a rate of $10 \% \mathrm{MBL} /$ minute and then held under tension for 30 minutes. Then the rope is unloaded to $10 \%$ MBL at the same load rate. This is followed by 100 cycles of dynamic bedding-in at $0.25 \mathrm{~Hz}$ between $10 \%$ and $30 \%$ MBL. The dynamic rope stiffness is calculated at the end of the standard bedding-in sequence.

\subsubsection{Performance characterisation}

The axial stiffness characteristics, both quasi-static and dynamic, of the rope sample characterize its performance as implemented in Phase A.

To investigate the quasi-static load-extension characteristics of the rope, three load cycles were applied to the samples. The rope was loaded from $10 \%$ MBL to $30 \%$ at a rate of $5 \% \mathrm{MBL} / \mathrm{min}$. The load was then held for 30 minutes and the rope unloaded at the same rate to $10 \% \mathrm{MBL}$ where it was held for 30 minutes before the next cycle.

Dynamic stiffness is representative of the near-linear behaviour of synthetic ropes observed under cycling due to wave action (ISO, 2007). To quantify the dynamic stiffness of the rope, three load ranges are specified in the order of $10 \%$ of the MBL. At each load range, 100 cycles with a period of $15 \mathrm{~s}$ are applied. For the RRT, the chosen load ranges are 20- 
30\% MBL, 30-40\% MBL and 40-50\% MBL, respectively.

\subsubsection{Strength characterisation}

Load to failure allows for the verification of the breaking strength of the rope against the manufacturer's MBL specification. The break test was conducted as part of Phase B with a loading rate of $20 \%$ $\mathrm{MBL} / \mathrm{min}$.

While piston and gauge displacement were recorded for Phase A at both test facilities, the transducers were removed prior to implementing Phase B to avoid damage to them.

\subsubsection{Test regimes}

Three testing regimes were agreed between IFREMER and the University of Exeter to investigate whether the discussed standard is suitable for testing the polyester-polyolefin blend. These test regimes are listed in Table 3 and the estimated test duration is also noted.

Table 3. Description and duration of the test regimes employed for RRT of a fibre rope.

\begin{tabular}{|c|c|c|c|}
\hline $\begin{array}{l}\text { Test } \\
\text { regime }\end{array}$ & $\begin{array}{l}\text { Test } \\
\text { number }\end{array}$ & Test description & Duration \\
\hline 1 & $\begin{array}{l}\text { Test } 01 \\
\text { Test } 02 \\
\text { Test } 03\end{array}$ & Phase A $\rightarrow$ Phase B & $\begin{array}{l}5 \text { h } 50 \mathrm{~min} \\
20 \mathrm{~s}\end{array}$ \\
\hline 2 & Test 04 & $\begin{array}{l}\text { Phase A } \rightarrow \text { Rest } \rightarrow \\
\text { Phase B }\end{array}$ & $\begin{array}{l}23 \mathrm{~h} 50 \\
\min 20 \mathrm{~s}\end{array}$ \\
\hline 3 & Test 05 & $\begin{array}{l}\text { Phase A } \rightarrow \text { Rest } \rightarrow \\
\text { Phase A } \rightarrow \text { Phase B }\end{array}$ & $\begin{array}{l}34 \mathrm{~h} 50 \\
\min 20 \mathrm{~s}\end{array}$ \\
\hline
\end{tabular}

Test regime 1, where Phase $\mathrm{A}$ is immediately followed by Phase B, had three repeats (Test 01, Test 02 and Test 03) at each facility. These repeats provide a quantifiable margin for estimating the breaking strength of the rope relative to the nominal MBL. Test regime 2 (Test 04) involved a rest period of at least 18 hours before Phase B is implemented. Test regime 3 (Test 05) involved a repeated implementation of Phase A on the same sample after the rest period, followed by Phase B. Only one sample was tested under Test Regimes 2 and 3 at each facility.

For the rest periods in Test Regimes 2 and 3, the samples were unloaded overnight by detaching them at one end to fully remove the load, but they were left submerged at DMaC and kept being wetted throughout the rest period at IFREMER.

\subsection{Stiffness calculations}

Two elongation measurements are recorded for Phase A of each test, namely, piston displacement and changes in gauge length. However, since the piston displacement includes the displacements of splices and end loops it does not provide accurate stiffness values. Therefore, gauge measurements are used for determining stiffness parameters of the samples for steps in Phase A. For Phase B, only piston displacement measurements are available for characterising sample elongation since the transducer is removed for the break test.

The resulting stiffness for the tests is indicated by the relationship between the change in applied load and resulting elongation (measured by changes in gauge length) based on the below equation.

$\mathrm{K}=\frac{\Delta F}{\frac{e}{L_{R}} \times 100 \%}$

Where $K(\mathrm{kN} / \%)$ is the stiffness indicator, $F(\mathrm{kN})$ is the force and $e(\mathrm{~m})$ is the sample elongation, respectively.

The method to determine elongation measurements varies between $\mathrm{DMaC}$ (one transducer) and IFREMER (two transducers) due to the different instrumentation. The elongation at both facilities can be calculated by Equation 2:

$e=L_{G}-L_{R}$

where, $L_{G}(\mathrm{~m})$ is the final gauge length.

\subsubsection{End of bedding-in}

The stiffness at the end of bedding-in can be calculated as the slope of a linear regression of the forcestrain data points measured during the last five cycles in the $10-30 \%$ MBL load range. Figure 2 shows this regression plot for Test 01 at DMaC.

\subsubsection{Quasi-static stiffness}

Stiffness values are calculated for the first, second and third quasi-static loading cycles of the samples. These are defined as the change in load divided by the change in strain between the initial strain at $10 \%$ MBL just before loading and the maximum final

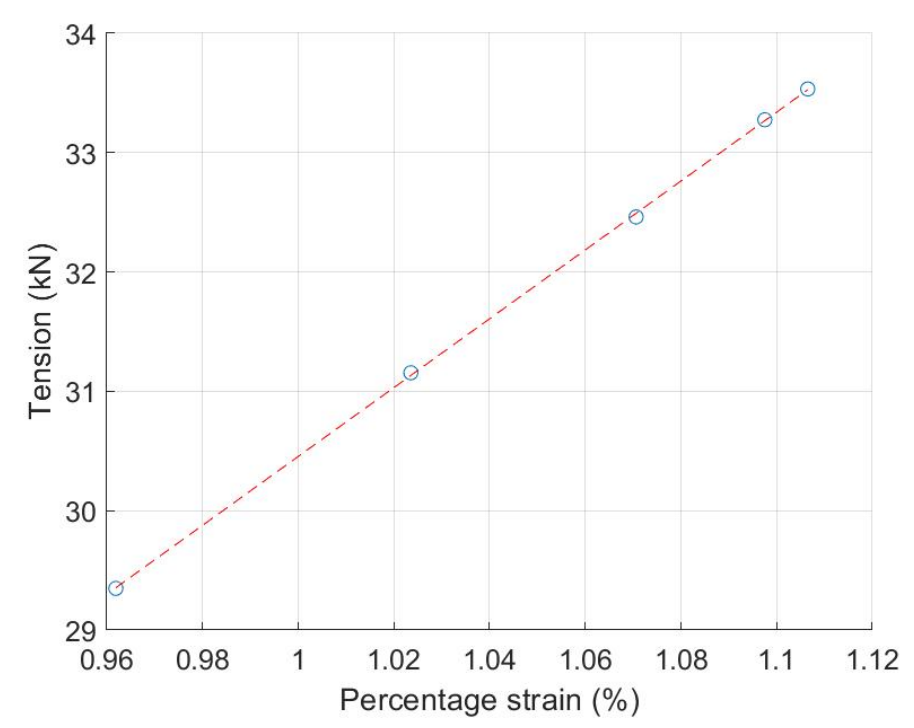

Figure 2. Regression plot for calculating stiffness at the end of dynamic bedding-in for Test 01 at DMaC. 
strain after 30 minutes at the higher $30 \%$ load just before unloading. The stiffness of the first of the 3 quasi-static cycles is not included in the calculation, as it is affected by the bedding-in cycles and the rope has not reached an equilibrium value before reloading starts.

\subsubsection{Dynamic stiffness}

The final set of stiffness values measured was the dynamic stiffness, measured at three load levels for cycles with a period of 15 seconds. The dynamic stiffness for each load range is defined as the slope of all the force-strain pairs recorded during the last five loading cycles for the respective load range, as shown for Test 01 at DMaC in Figure 3.

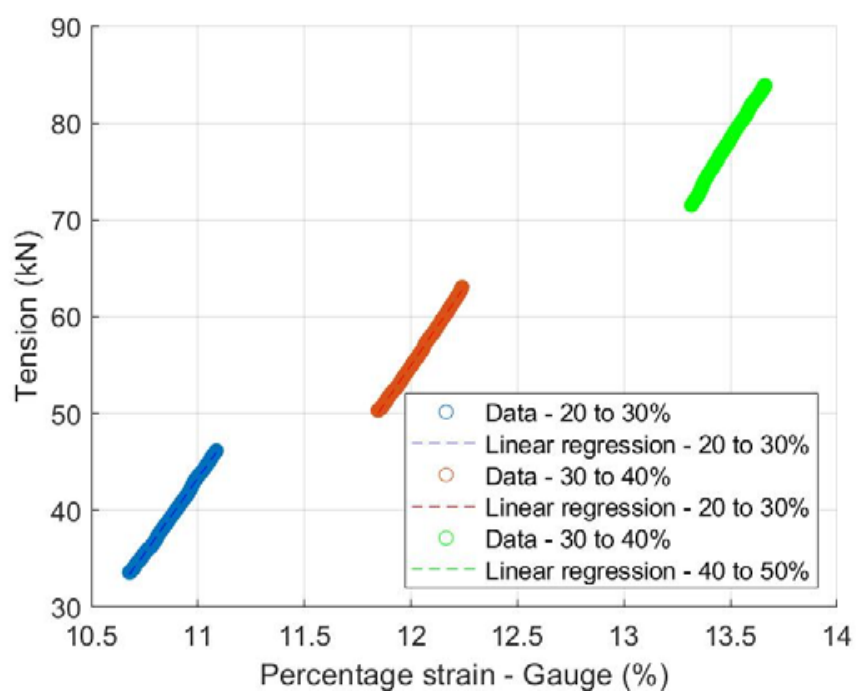

Figure 3. Regression plots for calculating dynamic stiffness at three load ranges (20-30\% MBL, 30-40\% MBL and 40$50 \%$ MBL) for Test 01 at DMaC.

\section{RESULTS}

Results of the RRT campaign, comparative implementation of various test regimes and characterization of the polyester-polyolefin blend are presented in this section.

\subsection{Representative load and elongation time series}

The same load sequence (Figure 4a) is applied to all samples to characterize stiffness in Phase A at both test facilities based on the nominal MBL of $168 \mathrm{kN}$. The steps of ISO 18692:2007(E) tabulated in Table 2 can be identified.

The resulting elongation time series for Test 01 at $\mathrm{DMaC}$ (Figure 4b) shows that the sample displays permanent elongation due to the alignment of the filaments and stabilization of the eye splices.

Time series for the Phase B (load-to-failure test) of Test 01 at DMaC is shown in Figure 4c. This time series displays the achieved break load of the sample at $127 \%$ of the nominal MBL.

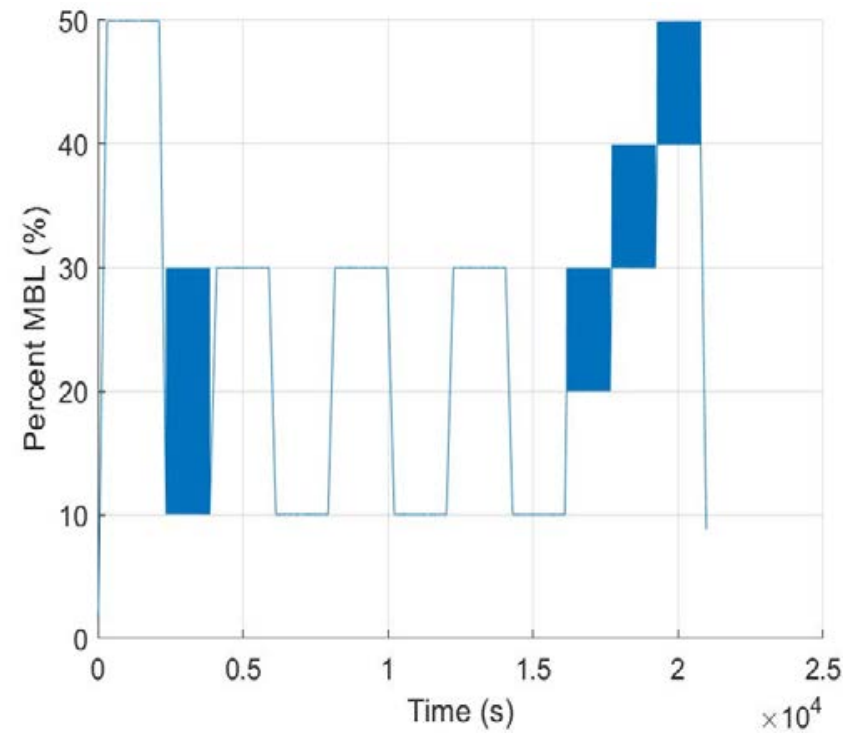

(a) Phase A load time series for all samples

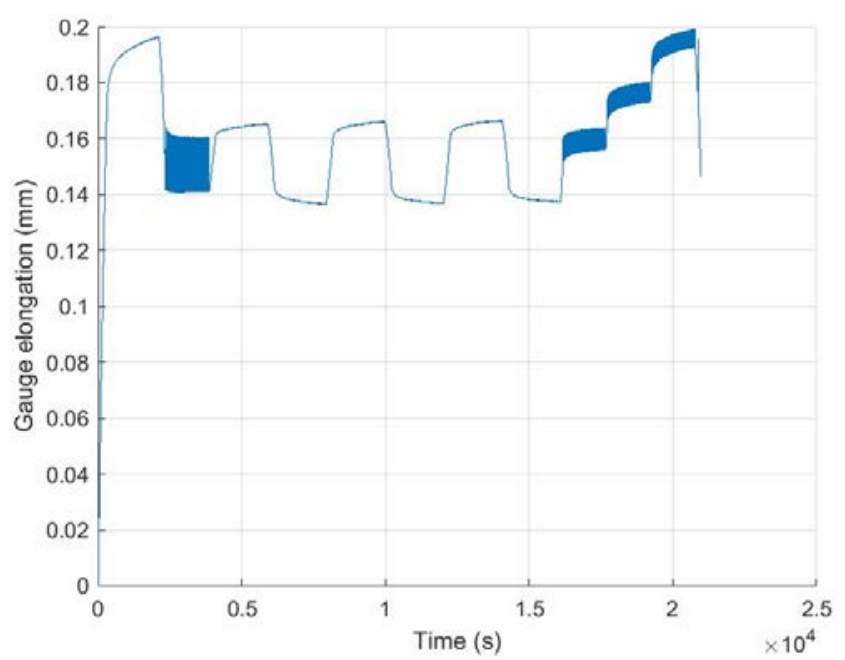

(b) Elongation time series for Test 01 at $\mathrm{DMaC}$

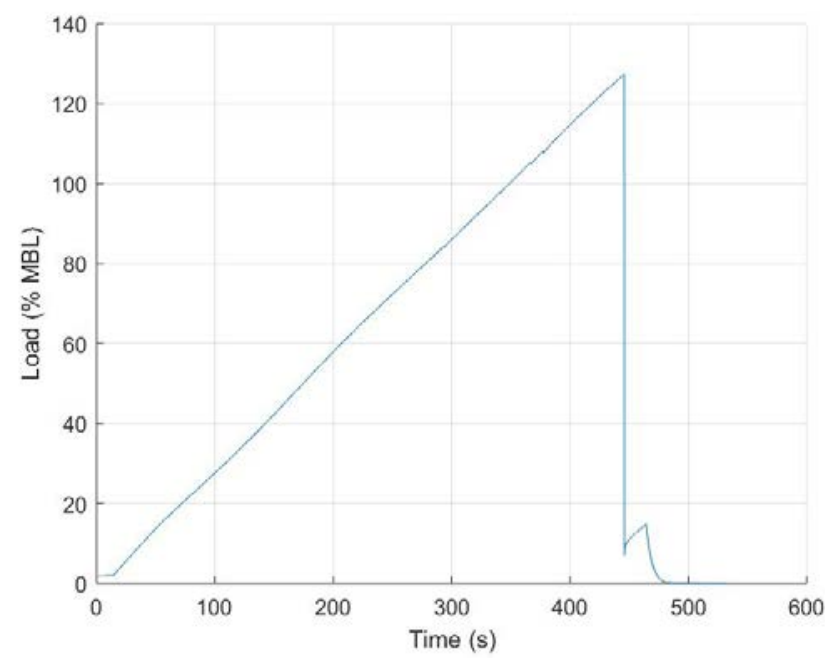

(c) Phase B load time series for Test 01 (DMaC)

Figure 4. Representative (a) load and (b) elongation time series for Phase A and (c) load time series for Phase B. 


\subsection{Comparison of RRT facilities}

A comparison between RRT results at DMaC and IFREMER is conducted for the following parameters:

- $\quad$ End of bedding-in stiffness

- Quasi-static stiffness

- Dynamic stiffness

- Load-to-failure test

For Test 05 at each facility, two stiffness values are shown. Test 05a represents the first implementation of Phase A and Test 05b presents the stiffness values for the second implementation of Phase A after being left unloaded overnight.

\subsubsection{End of bedding-in stiffness}

The stiffness of the various test specimens after the dynamic bedding-in is presented in Figure 5a. The mean value for the bedding-in stiffness, $28.9 \mathrm{kN} / \%$, is calculated from Test 01 to Test 05a (Test 05b is not included).

It can be observed that the stiffness at both facilities is within $12.5 \%$ of the mean, including the measurement in Test 05b. Furthermore, the variance of the measurements at IFREMER is lower than at $\mathrm{DMaC}$

\subsubsection{Quasi-static stiffness}

Due to a programming error and wire transducer malfunction, quasi-static stiffness results from Test 01 at IFREMER are not included in the stiffness calculation.

Figure 5b shows the average stiffness of the various test specimens for the last two quasi-static cycles. The mean value for the quasi-static stiffness, 17.4 $\mathrm{kN} / \%$, is calculated from Test 01 to Test 05a for $\mathrm{DMaC}$ and Test 02 to Test 05a for IFREMER. Test 05b is not included for both facilities, whereas, Test 01 is not included for IFREMER.

Good agreement is found between quasi-static stiffness at both facilities. The measurements are within $5 \%$ of the mean stiffness value. The variance in quasi-static stiffness is lower at IFREMER relative to $\mathrm{DMaC}$ samples.

\subsubsection{Dynamic loading}

Figure $5 c$ shows the stiffness of the various test specimens for the three specified dynamic load ranges (20 - 30\% MBL, 30 - 40\% MBL and $40-50 \%$ MBL). The mean values for the dynamic stiffness are $31.4 \mathrm{kN} / \%, 34.5 \mathrm{kN} / \%$ and $37.5 \mathrm{kN} / \%$, respectively. These are calculated from Test 01 to Test 05 a for both DMaC and IFREMER (Test 05b is not included).

In this case, the stiffness at IFREMER is within $15 \%$ of the mean relative to $10 \%$ for $\mathrm{DMaC}$ at all applied cyclic load ranges. Contrary to quasi-static stiffness, variance in the dynamic stiffness measurements at $\mathrm{DMaC}$ is lower than at IFREMER.

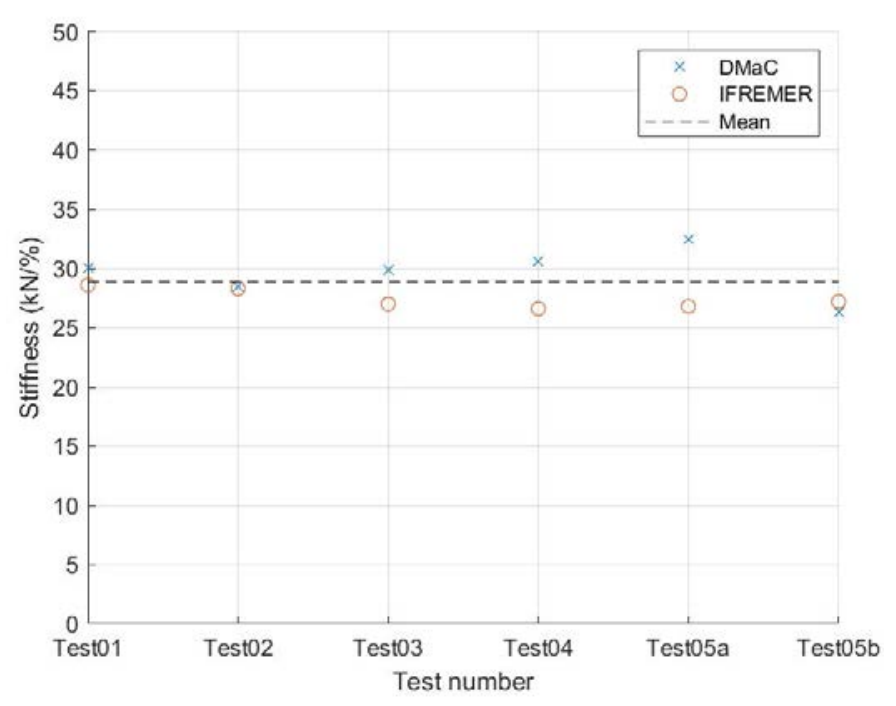

(a) End of bedding-in stiffness

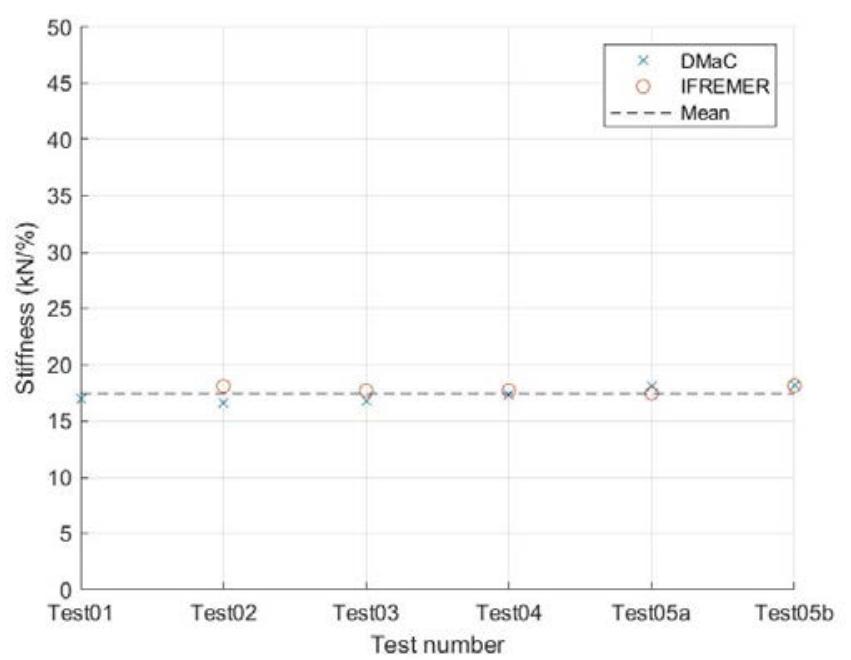

(b) Quasi-static stiffness

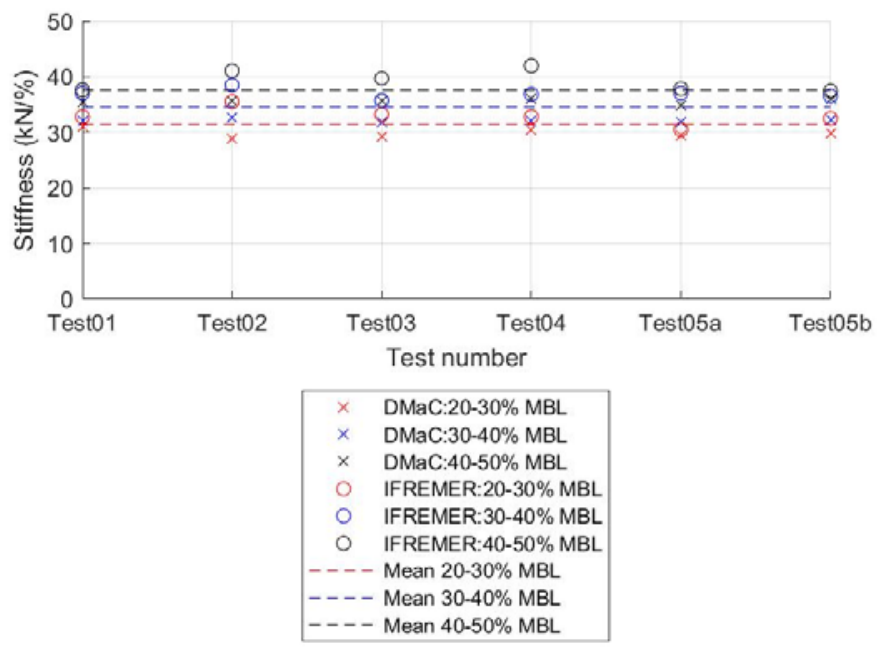

(c) Dynamic stiffness

Figure 5. RRT stiffness results (a) at the end of bedding-in, (b) quasi-static loading and (c) dynamic loading for DMaC and IFREMER. 


\subsubsection{Break test}

Figure 6a. shows the break load of the various test specimens at each test facility. The mean value for the break load, $204 \mathrm{kN}$ (121 \% MBL), is calculated from Test 01 to Test 03 for DMaC and IFREMER (Test 04 and Test 05 are not included). The break loads for Test04 do not provide an accurate RRT comparison since the sample at $\mathrm{DMaC}$ did not break once the rest period ended. Test-to-failure was attempted thrice; however, the sample was too elastic to break. It was then left to dry and the test-to-failure was conducted after a further 24 hours to break the rope.

It can be seen that all samples at both facilities exceeded the break load specified by the manufacturer, some by up to 35\%. Variance for break test measurements is lower at DMaC than that at IFREMER.

At $\mathrm{DMaC}$, one strand failed for all ropes at or near the end of splice (Figure 6b.). Similarly, for IFREMER the failure was due to one strand breaking at or near the splice except Test 02 (Figure 6c.) where all 3 strands broke in the splice.

The broken ropes showed an unusual failure aspect, presumably related to the inclusion of two different fibre types with different characteristics. In the region of final failure there was a "furring" of the rope, as shown in Figure 6d. Further investigation of this failure mode may be of interest.

\subsection{Suitability of the applied test standard}

The bedding-in methodology recommended by ISO 18692:2007(E) for pure polyester ropes was found to be sufficient for the bedding-in the relatively more elastic polyolefin blend as shown in the elongation time series in Figure 4b.

To further investigate the suitability of the standard, three test regimes were tested at each facility.

Based on the similarity of implementing Phase A on all five samples, statistical parameters for Phase A (dynamic bedding-in, quasi-static loading and dynamic loading) are calculated based on measurements from all five samples. It must be noted that the measurements from the second implementation of Phase A in Test 05 are not included. Additionally, the quasistatic bedding-in of the specimen in Test 01 from IFREMER is not included due to the programming error that occurred whilst performing the test.

As the break test, Phase B, is conducted in similar fashion for Test 01, Test 02 and Test 03 only, therefore, only three tests are used to calculate the mean break load of the samples at each facility. Also, it must be noted that the results for the break test of Specimen 04 are not comparable between test facilities as the rest period and conditions were not comparable (refer to Section 3.2).

Figure 7 shows the mean stiffness properties and error bars for the polyester and polyolefin blend rope for various stages in the implemented load regime. 
It can be observed that both facilities show some variability in stiffness values, occuring for the break test. Mean stiffness values at IFREMER are higher than at DMaC except for that at the end of dynamic bedding in. The standard deviation between samples is higher at DMaC for all stages except the three dynamic loading ranges.

Figure 7 shows that the second implementation of Phase A in Test 05 (referred to as Test 05b) leads to higher stiffness values for quasi-static and dynamic loading at DMaC. At IFREMER, it is observed that while the quasi-static stiffness of the sample is higher than the mean, dynamic stiffness values are lower than the mean of five samples undergoing Phase A once.

\subsection{Characterisation of rope material}

The mean dynamic stiffness values of the polyester-polyolefin rope specimens can be compared to publically available stiffness parameters of commonly used synthetic materials such as $100 \%$ polyester and 100\% Polyamide 6 (nylon) ropes. Such values can be expressed in normalized form by dividing the stiffness by the MBL and converting to strain rather than percentage strain. Some typical values are given in Table 4 below.

Table 4. Comparison of dynamic stiffness of sample with pure polyester and nylon ropes.

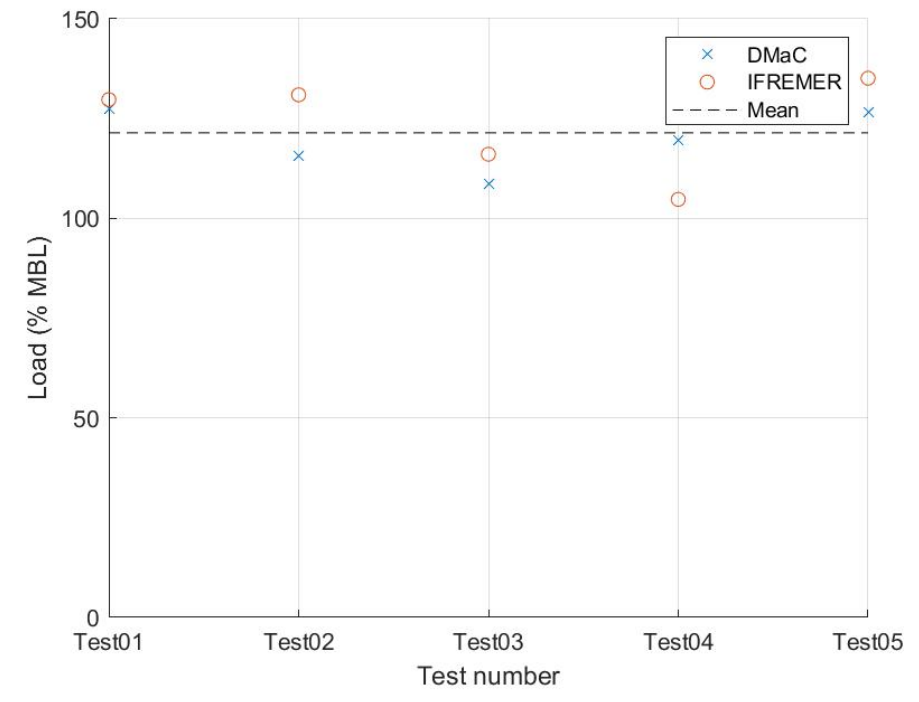

(a) RRT strength test at DMaC and IFREMER

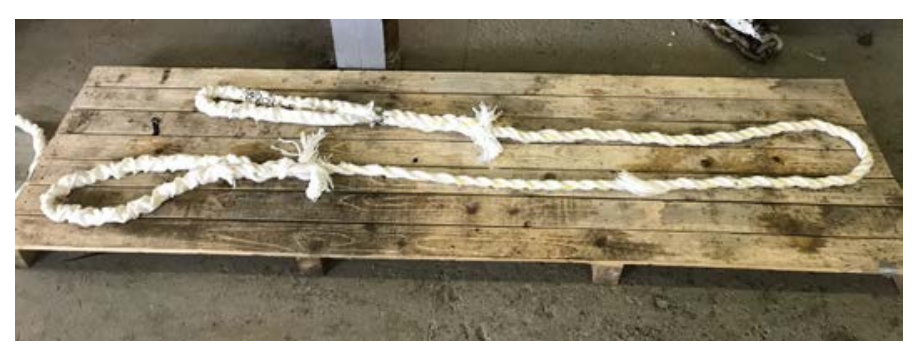

(b) 1 broken strand near end of splice (Test 02) at DMaC

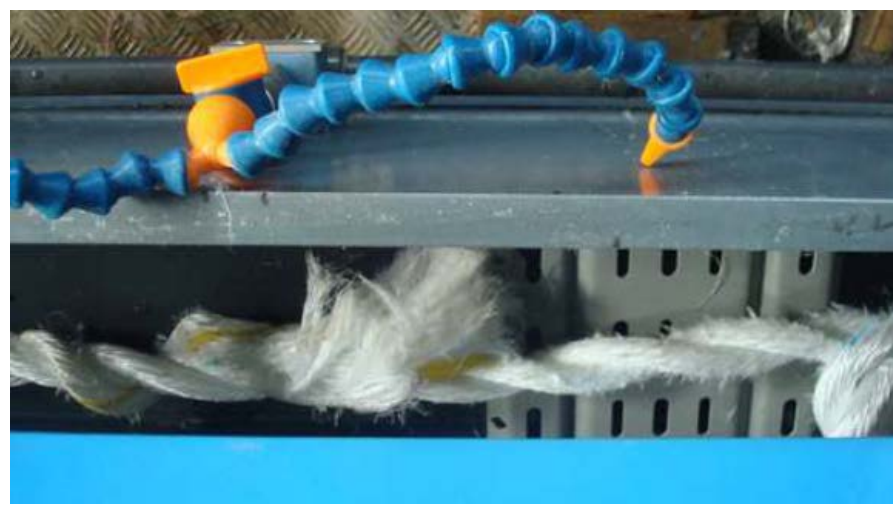

(c) 3 broken strands at end of eye (Test 02) at IFREMER

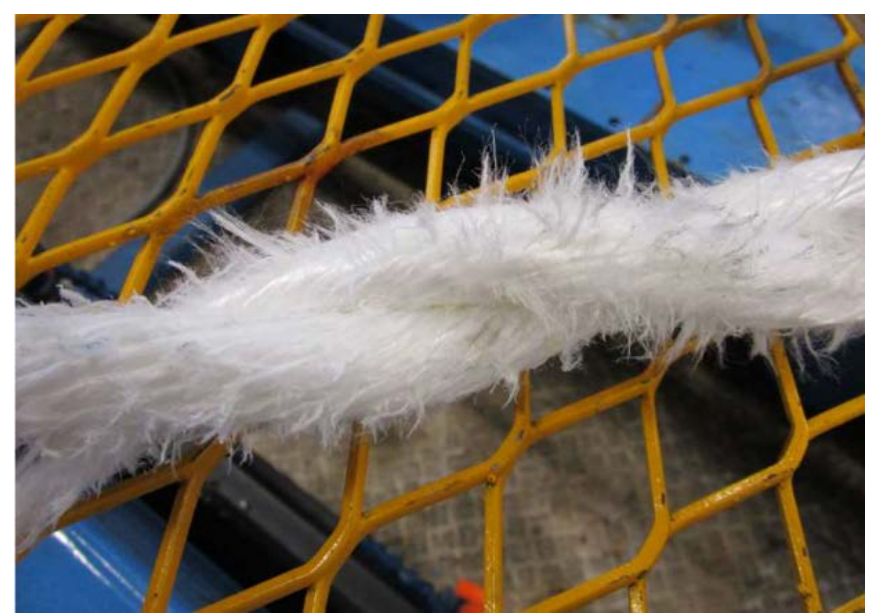

(d) Furring of rope near failure zone

Figure 6. Phase B (a) RRT campaign results at DMaC and IFREMER, (b) dominant and (c) unusual failure modes and (d) unusual furring near failure zone. 


\begin{tabular}{lccl}
\hline * (Francois et al., 2010), ** Internal IFREMER data \\
\hline Material & $20-$ & $30-40 \%$ & $40-50 \%$ \\
& $30 \%$ & & \\
\hline Polyester* & 27 & 30 & 33 \\
PP+PE & 19.6 & 22 & 23.9 \\
(IFREMER) & & & \\
PP+PE (DMaC) & 17.77 & 19.12 & 21.15 \\
Polyamide 6** & - & 10 & 13 \\
\hline
\end{tabular}

The comparison of $100 \%$ nylon and $100 \%$ polyester with values for the polyester-polyolefin blend tested here shows that the dynamic stiffness of this hybrid rope is lower than that of $100 \%$ polyester but higher than that of nylon.

\section{DISCUSSION}

The RRT campaign for synthetic fibre rope testing conducted at DMaC and IFREMER has provided useful outcomes to increases confidence in the test activities at both facilities. The various stiffness and strength measurements generally showed a good agreement ( $<15 \%$ difference from mean) between both facilities. Data variance can be used as an indicator for the precision of the conducted tests at each facility. IFREMER displayed a higher precision for stiffness calculations at the end of bedding-in and quasi-static loading, whereas, DMaC had higher repeatability for dynamic loading and break testing.

A possible reason for the variation in recorded stiffness and strength measurements may be due to the resplicing of the ropes. ISO 18692:2007(E) (ISO, 2007) identifies a change in the design or method of terminations as a source of modification of the rope properties.

The test plan adopted from guidance for pure polyester ropes was found to be suitable for the polyester- polyolefin blend. The test sequence included an initial bedding-in sequence and subsequent quasistatic and dynamic loading followed by load-to -failure. The cycling period of the dynamic loading does not provide a comprehensive analysis of the environmental conditions faced by an MRE device. Further analysis should be conducted at higher frequency to account for the effect of dynamic wave conditions on synthetic rope performance.

Employing divergent test regimes did not significantly influence the stiffness or strength results. ISO 18692:2007(E) (ISO, 2007) advises that after the initial bedding-in, further load-elongation measurements that may be performed will not affect the break load of the rope. This was confirmed by Test Regime 2 and 3, however, further tests should be conducted at

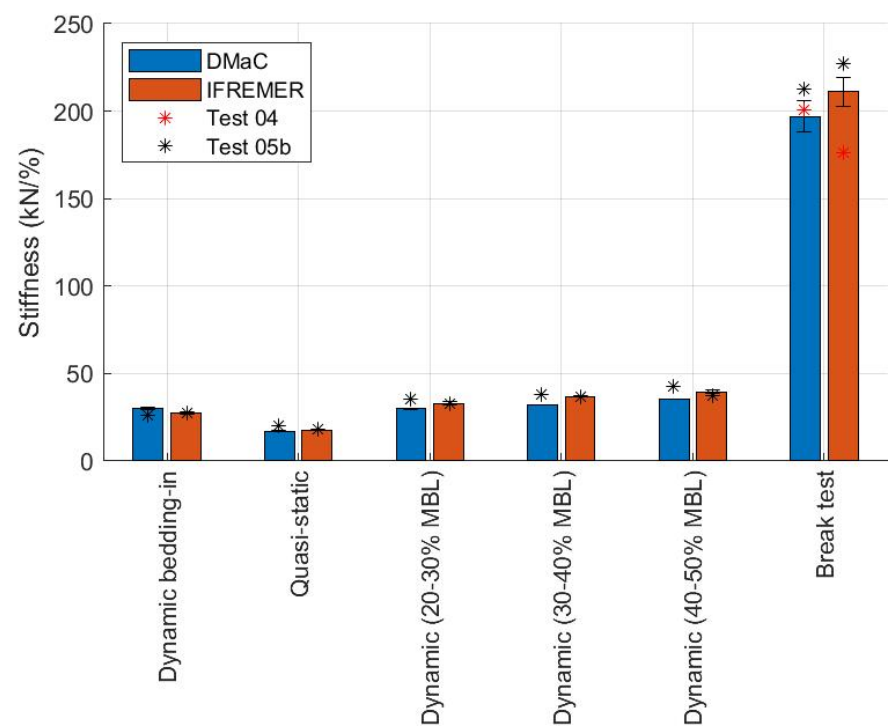

both facilities to ensure the reproducibility of these test regimes.

The test specimen was a hawser laid rope with polyester wound round polyolefin. Unlike nylon, both polyester and polyolefin fibres have negligible water absorption and are not expected to lose strength when used for offshore applications. The higher compliance of the polyolefin is seen to overcome the stiffness of the polyester and may allow this blend to show improved shock load absorption. However, further investigation of fatigue life and individual yarn stiffness to model the rope structure for improved understanding of its behaviour.

\section{CONCLUSION}

The RRT campaign involved planning, conducting and analyzing the results of the inter-facility study to quantify facility-specific influences on tests conducted at DMaC and IFREMER. In addition to increasing confidence in the performance of the facilities, outcomes of this joint RRT activity will further improve the service provided by the testing infrastructures. These outcomes may be used to validate the standardisation techniques for mooring component testing in MRE and inform technical working group activities. By delivering quality reference data sets for open access, this paper lends itself to inform future research into fibre rope testing for MRE applications.

\section{ACKNOWLEDGEMENT}

The collaborative project between University of Exeter and IFREMER leading to these results has received funding from the European Union Horizon 2020 Framework Programme (H2020) under grant agreement no 731084 of the Marine Renewables Infrastructure Network for emerging Energy Technologies (MaRINET2) project. 


\section{REFERENCES}

ClassNK. (2012). Guidelines for offshore floating wind turbine structures.

Davies, P., Weller, S. D., Johanning, L., \& Banfield, S. J. (2014). A review of synthetic fiber moorings for marine energy applications. 5th International Conference on Ocean Energy, November, 1-6.

Davies, P., Weller, S., \& Johanning, L. (2012). D3.5.1 MERiFIC - Testing of synthetic fibre ropes.

Det Norske Veritas (DNV). (2008). Certification of tidal and wave energy converters (DNV-OSS312, Amended April 2012).

DNV-GL. (2015). DNVGL-ST-0164: Tidal turbines.

DNV GL. (2018). DNVGL-ST-0119: Floating wind turbine structures.

Flory, JF; Banfield, SJ; Ridge, I. (2016). Mooring systems for marine energy converters. OCEANS

Figure 7. Comparison of break stiffness of Test 04 and all stiff-

ness values for Test $05 b$ with mean stiffness at each facility. '16 Monterey MTS.

Francois, M., Davies, P., Grosjean, F., Legerstee, F., \& others. (2010). Modelling fiber rope loadelongation properties-Polyester and other fibers. Offshore Technology Conference.

Gordelier, T. J. (2016). Enhancing wave energy developments through mooring system reliability assessment. July.

IEC. (2013). Marine energy - Wave, tidal and other water current converters - Part 10: The assessment of mooring system for marine energy converters (MECs) IEC/TS 62600-10. In preparation.

ISO. (2007). Fibre ropes for offshore stationkeeping - Polyester ISO 18692.

Khalid, F., Arini, N. R., \& Johanning, L. (2019). OPERA - Recommendations for WEC mooring guidelines and standards.

Lankhorst Ropes. (2020). EUROFLEX® 3 STRAND. https://www.lankhorstropes.com/products/eurof lex-3-strand

Ridge, I. M. L., Banfield, S. J., \& Mackay, J. (2010). Nylon fibre rope moorings for wave energy converters. MTS/IEEE Seattle, OCEANS 2010, June 2015.

The Carbon Trust. (2011). Accelerating Marine Energy. In The potential for cost reduction insights from the Carbon Trust Marine Energy Accelerator.

Weller, S. D., Johanning, L., Davies, P., \& Banfield, S. J. (2015). Synthetic mooring ropes for marine renewable energy applications. Renewable Energy, 83, 1268-1278. 